\title{
Macrophage colony-stimulating factor (M-CSF) intermediately participating in the process of \\ luteinizing hormone-induced natriuretic peptide receptor 2 (NPR2) decrease and oocyte meiotic resumption

\author{
Wenchao Sun, Chang Liu, Ying Feng, Guangchao Zhuo, Wenjing Zhou, Xiaoyang Fei, and \\ Zhifen Zhang
} Center of Reductive Medicine, Hangzhou Obstetrics and Gynecology Hospital, Nanjing Medical University, Hangzhou, China

\section{Problem statement: Luteinizing hormone (LH) regulation of the ligand natriuretic peptide precursor type $C$ and its receptor natriuretic peptide receptor 2 (NPR2) is critical for oocyte maturation. But the mechanism is not fully understood. Macrophage colony-stimulating factor (M-CSF) has recently been demonstrated to be involved in the processes of oocyte maturation and ovulation.}

Methods: We explored the effect of M-CSF in the LH-induced regulation of oocyte meiosis using immature female C57BL/6 mice. Mice were injected i.p. with $5 \mathrm{IU}$ of equine chorionic gonadotropin (eCG) to stimulate follicle development. After 44$48 \mathrm{~h}$, the eCG-stimulated mice were next injected i.p. with an ovulatory dose of $5 \mathrm{IU}$ human chorionic gonadotropin (hCG). Ovaries were excised at selected times. Pre-ovulatory follicles (POFs) and cumulus-oocyte complexes were cultured in different medium. Immunohistochemical analysis and quantitative realtime PCR analysis were used to assess the expression of $M$ CSF, M-CSF receptor (M-CSF-R), and NPR2. The presence of germinal vesicle breakdown (GVBD) was examined under a stereomicroscope to morphologically evaluate the meiosis resumption of oocytes.

Results: NPR2 was mainly expressed in cumulus cells of POFs, while M-CSF and M-CSF-R were expressed in both mural granulosa cells and cumulus cells. The levels of M-CSF/M-CSF$\mathrm{R}$ and NPR2 decreased within $4 \mathrm{~h}$ after hCG treatment. M-CSF not only reduced the expression of NPR2 mRNA via its receptor M-CSF-R, but also increased the proportion of GVBD of oocyte.

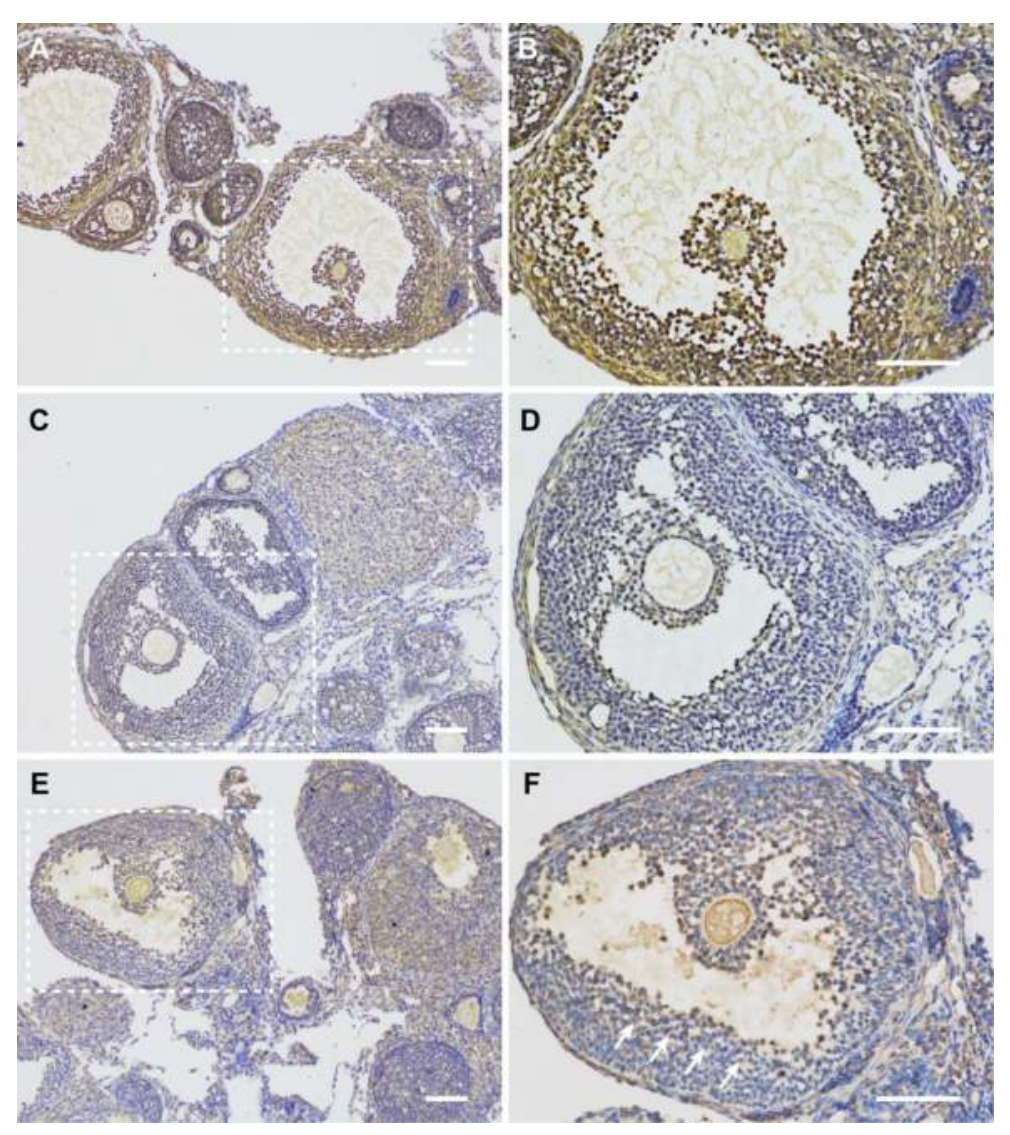

Figure 1. Expression patterns of M-CSF, M-CSF-R and NPR2 in the ovaries of eCG-stimulated mice. Localization of $M-C S F$ ( $A$ and $B)$, $M-C S F-R$ (C and D) and NPR2 (E and F) was analyzed using immunohistochemis try. White arrows, periantral mural GCs. Scale bar, 100 $\mu \mathrm{m}$.
Conclusion: M-CSF plays the role of an intermediate signal, inducing a vital decrease in NPR2 levels in cumulus cells, and regulates the process of $\mathrm{LH}$-induced meiotic resumption.
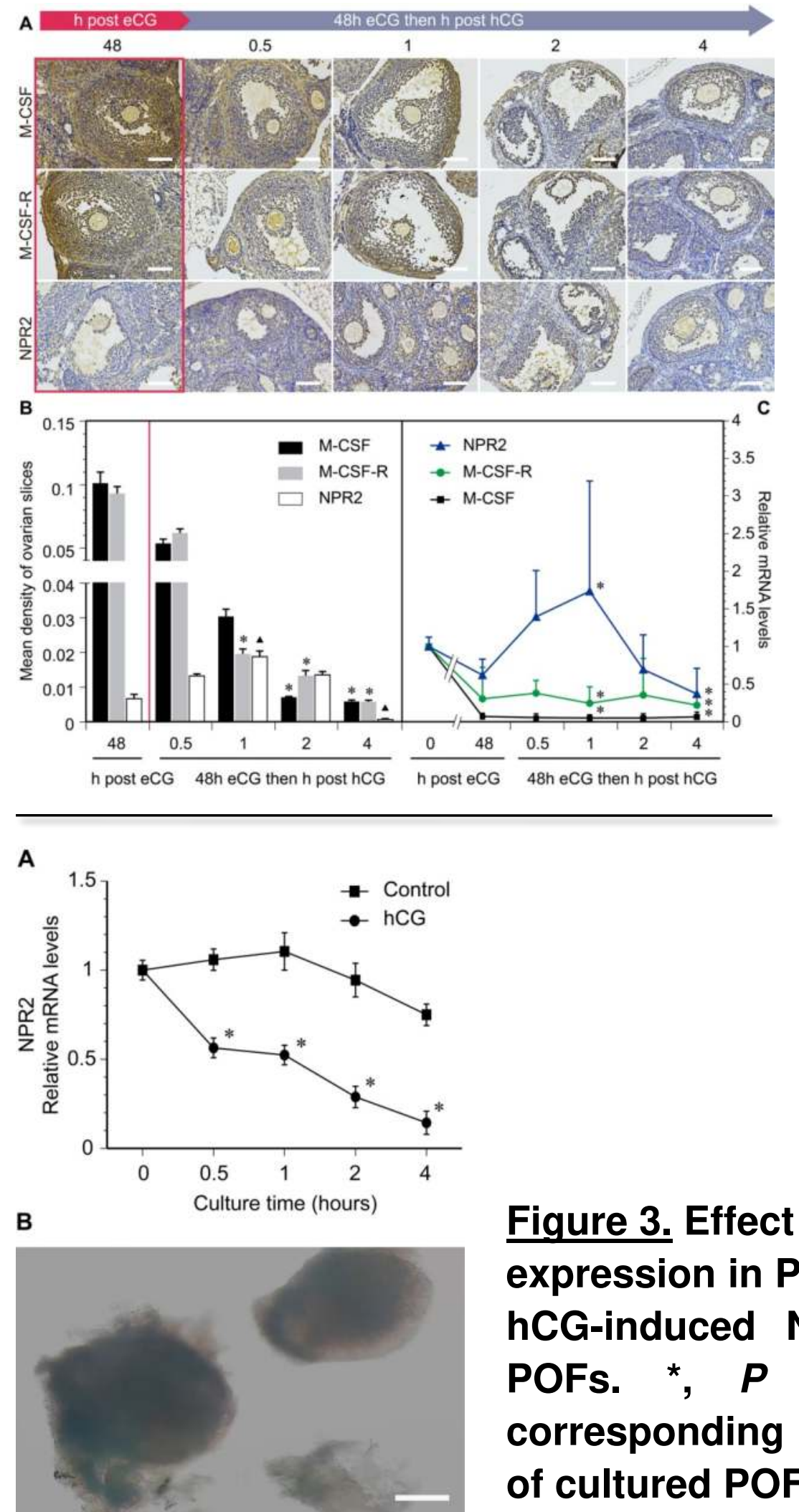

Figure 2. Gonadotropin control of M-CSF, MCSF-R and NPR2 expression in ovaries in vivo. (A) Localization of M-CSF, M-CSF-R and NPR2 in ovarian follicles shown with immunohistochemistry - Red box represents the time point of $48 \mathrm{~h}$ after eCG treatment. Scale bars, $100 \mu \mathrm{m}$. (B) Mean optical density of ovarian slides. (C) Expression of M-CSF, M-CSF-R and NPR2 mRNA by quantitative RT-PCR. * * $\boldsymbol{P}<.05$ compared with the corresponding control.

Figure 3. Effect of hCG on NPR2 mRNA expression in POFs. (A) The kinetics of hCG-induced NPR2 mRNA levels in POFs. *, $P<.05$ compared with corresponding control. (B) The image of cultured POFs. Scale bar, $100 \mu \mathrm{m}$.

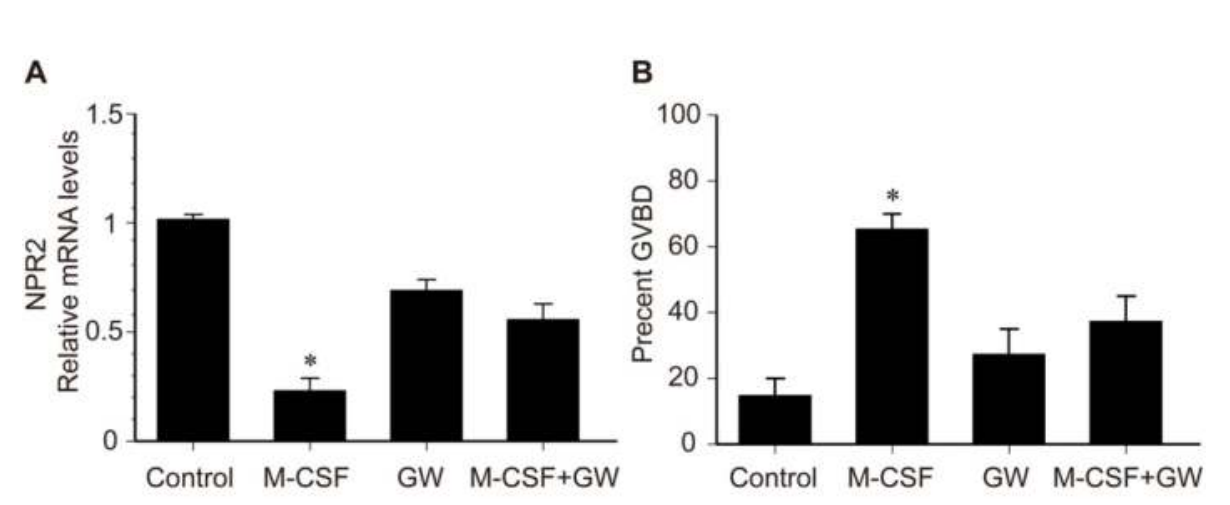

Figure 4. Effect of M-CSF on NPR2 mRNA expression and oocyte maturation. COCs isolated from eCG-stimulated mice were

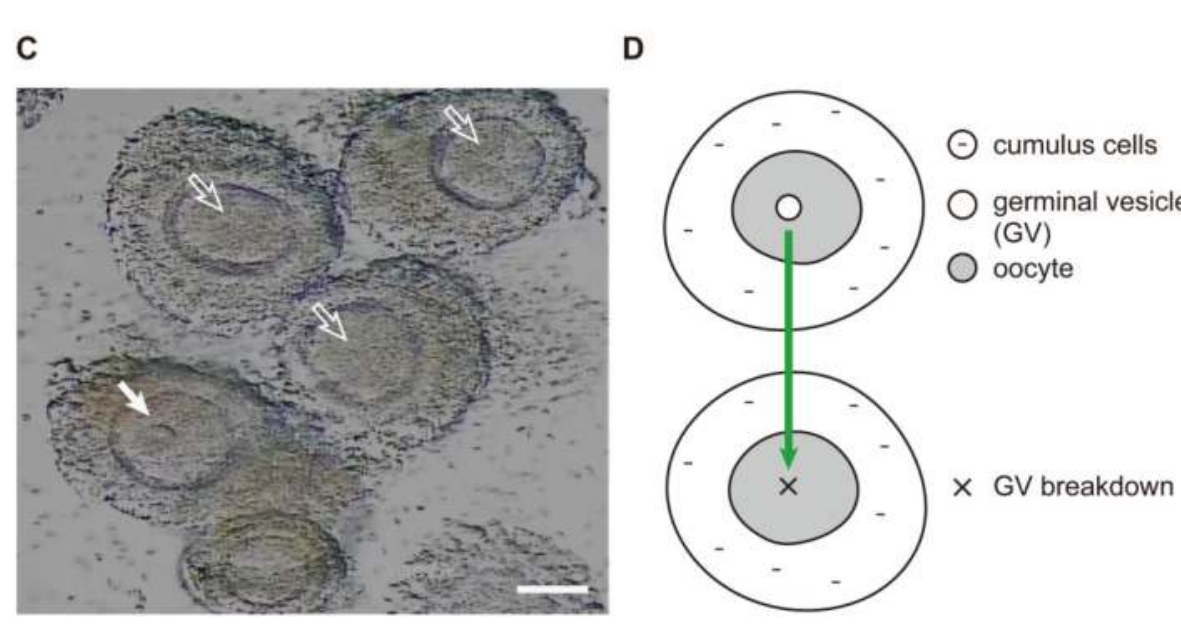
ultured in MEM-a in the existence of $30 \mathrm{nM}$ NPPC (control) added with $200 \mathrm{ng} / \mathrm{ml} \mathrm{M}$ CSF and/or $1 \mu \mathrm{mol} / \mathrm{L}$ GW2580 for $2 \mathrm{~h}$. 